\title{
Improvement of Resistive Switching Performance in Sulfur-Doped HfOx-Based RRAM
}

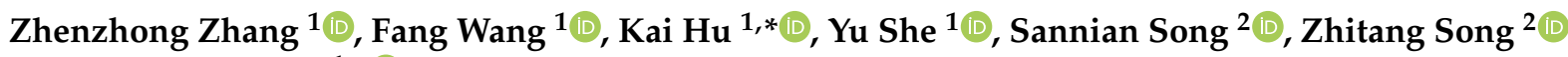 \\ and Kailiang Zhang $1, * \mathbb{B}$
}

1 Tianjin Key Laboratory of Film Electronic and Communication Devices, School of Electrical and Electronic Engineering, Tianjin University of Technology, Tianjin 300384, China; zhangzz_1128@163.com (Z.Z.); fwang75@163.com (F.W.); yushe_11@163.com (Y.S.)

2 State Key Laboratory of Functional Materials for Informatics, Shanghai Institute of Micro-System and Information Technology, Chinese Academy of Sciences, Shanghai 200050, China; songsannian@mail.sim.ac.cn (S.S.); ztsong@mail.sim.ac.cn (Z.S.)

* Correspondence: kay@email.tjut.edu.cn (K.H.); kailiang_zhang@163.com (K.Z.)

Citation: Zhang, Z.; Wang, F.; Hu, K.; She, Y.; Song, S.; Song, Z.; Zhang, K. Improvement of Resistive Switching Performance in Sulfur-Doped HfOx-Based RRAM. Materials 2021, 14, 3330. https://doi.org/10.3390/ ma14123330

Academic Editors: Antonio Di Bartolomeo and Christof Schneider

Received: 3 May 2021

Accepted: 14 June 2021

Published: 16 June 2021

Publisher's Note: MDPI stays neutral with regard to jurisdictional claims in published maps and institutional affiliations.

Copyright: () 2021 by the authors. Licensee MDPI, Basel, Switzerland. This article is an open access article distributed under the terms and conditions of the Creative Commons Attribution (CC BY) license (https:// creativecommons.org/licenses/by/ $4.0 /)$.

\begin{abstract}
In order to improve the electrical performance of resistive random access memory (RRAM) sulfur (S)-doping technology for HfOx-based RRAM is systematically investigated in this paper. HfOx films with different S-doping contents are achieved by atmospheric pressure chemical vapor deposition (APCVD) under a series of preparation temperatures. The effect of $\mathrm{S}$ on crystallinity, surface topography, element composition of HfOx thin films and resistive switching (RS) performance of HfOx-based devices are discussed. Compared with an undoped device, the $\mathrm{V}_{\mathrm{SET}} / \mathrm{V}_{\mathrm{RESET}}$ of the S-doped device with optimal S content ( $1.66 \mathrm{At} . \%)$ is reduced, and the compliance current (Icc) is limited from $1 \mathrm{~mA}$ to $100 \mu \mathrm{A}$. Moreover, it also has high uniformity of resistance and voltage, stable endurance, good retention characteristics, fast response speed (SET $6.25 \mu \mathrm{s} /$ RESET $7.50 \mu \mathrm{s})$ and low energy consumption (SET $9.08 \mathrm{~nJ} /$ RESET $6.72 \mathrm{~nJ}$ ). Based on X-ray photoelectron spectroscopy (XPS) data and fitting of the high/low resistance state (HRS/LRS) conduction behavior, a switching mechanism is considered to explain the formation and rupture of conductive filaments (CFs) composed of oxygen vacancies in undoped and S-doped HfOx-based devices. Doping by sulfur is proposed to introduce the appropriate concentration oxygen vacancies into HfOx film and suppress the random formation of $\mathrm{CFs}$ in HfOx-based device, and thus improve the performance of the TiN/HfOx/ITO device.
\end{abstract}

Keywords: HfOx-based RRAM; sulfur-doping; RS performances improvement; switching mechanism

\section{Introduction}

Resistive random access memory (RRAM), as the emerging non-volatile memory, has the potential to replace traditional NAND flash [1-3]. RRAM has some unique advantages: its simple structure, good scalability, low operating voltages, low energy consumption, fast reading and writing speed, high memory density and excellent compatibility $[4,5]$. As the excellent resistive medium layers of RRAM, binary-transition metal oxide (B-TMO) materials ( $\mathrm{HfOx}$ [6], $\mathrm{TiO}_{2}$ [7], $\mathrm{ZnO}$ [8], $\mathrm{ZrO}_{2}$ [9], $\mathrm{TaOx}$ [10], WOx [11], etc.) have been objects of research interest [4,12]. Among them, the high-k ( 21) HfOx is considered one of the most promising materials for commercial applications due to its overall performances in terms of reliability, including endurance, retention and operation stability, etc. [13,14]. However, it is still difficult to comprehensively improve device performances; for example, its low energy consumption and high cycle-to-cycle uniformity. Researchers have proposed some solutions to solve the existing problems for RRAM: ion doping $[9,12,15,16]$, double-layer structure [17-19], electrode engineering [20,21], etc. Among these methods, ion doping is considered an effective method to improve device uniformity, energy consumption and other performances $[15,16,22]$. There have recent related studies on sulfur 
(S)-based resistive switching devices. Jang et al. [23] reported the $\mathrm{Ag}_{2} \mathrm{~S}$ based RRAM with improved endurance and retention using different electrode materials. Li et al. [24] reported a $\mathrm{HfS}_{2}$ film memory device based on density functional theory calculations. Zheng et al. [25] proposed an electrode annealing method to improve the RS properties in $\mathrm{PbS}$ micro/nanowire-based devices for RRAM.

In our work, S-doped HfOx RRAM devices are fabricated using atmospheric pressure chemical vapor deposition (APCVD) treatment. The resistive switching performance of the HfOx-based devices are improved and the physical characteristics of the S-doped HfOx films are investigated. The $S$ dopant is suggested to introduce an appropriate concentration of oxygen vacancies into $\mathrm{HfOx}$ film and suppress the random formation of $\mathrm{CFs}$ in the HfOx-based device.

\section{Experimental Section}

A layer of 50-nm TiN film as the bottom electrode (BE) was first deposited on a cleaned Si substrate by direct current (DC) sputtering with Ti as the metal target $(99.99 \%)$ under an $\mathrm{Ar} / \mathrm{N}_{2}$ atmosphere. A 7-nm thick HfOx thin film was sputtered on TiN with a $\mathrm{HfO}_{2}$ ceramic target $(99.99 \%)$ under an $\mathrm{Ar} / \mathrm{O}_{2}$ atmosphere. Then, the $\mathrm{HfOx}$ film was treated by APCVD for 30 min under sulfur atmosphere at different temperatures of $400{ }^{\circ} \mathrm{C}$, $500{ }^{\circ} \mathrm{C}, 600{ }^{\circ} \mathrm{C}$. The Ar flow rate was maintained at $30 \mathrm{sccm}$ during the whole process, as shown in Figure 1a. After that, a 200-nm thick top electrode (TE) was deposited by the indium tin oxide (ITO) ceramic target $(99.99 \%)$ with a $300-\mu \mathrm{m}$ diameter metal mask. Figure 1b,c shows the structure of TiN/S:HfOx/ITO and an optical micrograph of the device, respectively. The chemical compositions of the undoped and S-doped HfOx films were characterized by XPS (Scientific Escalab 250Xi, Waltham, MA, USA). The film morphology was observed via atomic force microscopy (AFM, Bruker Dimension Icon, Santa Clara, CA). The electrical performances of undoped and S-doped HfOx-based devices were measured using a semiconductor parameter analyzer (Agilent B1500A, Santa Clara, CA, USA). For the electronic measurement of samples, a bias voltage was applied to the TE of ITO while the BE of TiN was grounded.
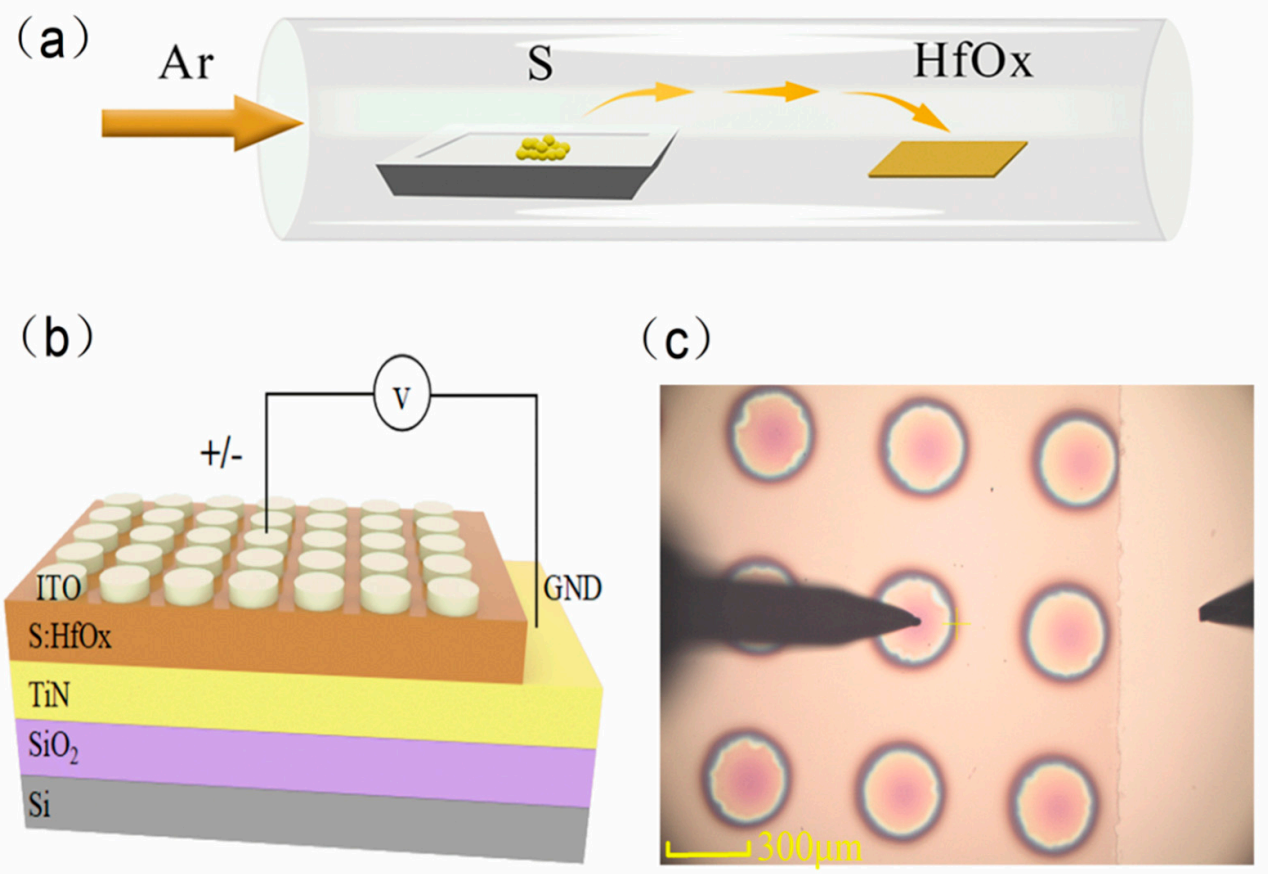

Figure 1. (a) Schematic diagram of two-temperature zone tube furnace. (b) Structure of TiN/S:HfOx/ITO for the S-doped device (The left probe contacts the ITO electrode and the right probe contacts GND). (c) Optical micrograph of the device. 


\section{Results and Discussions}

Figure 2a shows the typical bipolar resistive switching I-V curves of undoped and Sdoped HfOx devices at an Icc of $1 \mathrm{~mA}$. The electrical forming process and the 10 continuous $\mathrm{I}-\mathrm{V}$ cycles of each device are shown in Figure S1 of the Supplementary Materials. For ease of description, the undoped and S-doped $400{ }^{\circ} \mathrm{C}, 500{ }^{\circ} \mathrm{C}$, and $600^{\circ} \mathrm{C}$ devices are named D1, D2, D3, and D4, respectively. Compared with the D1, the RESET behavior of D2 and D3 is formed to be sharp gradually. More importantly, the Icc of the D3 device can be limited at $100 \mu \mathrm{A}$, as shown in Figure $2 \mathrm{~b}$, and the $\mathrm{V}_{\mathrm{SET}} / \mathrm{V}_{\mathrm{RESET}}$ of $\mathrm{D} 3$ is $+0.11 \mathrm{~V} /-0.15 \mathrm{~V}$, which will contribute to a low power property. In particular, the D2 device has a slight switching characteristic tendency at $100 \mu \mathrm{A}$, and D3 can only switch at $1 \mathrm{~mA}$, as shown in Figure S2. The results show that S-doping plays an important role in the decrease in device switching current. Interestingly, the D4 device failed to exhibit the typical resistive behavior, as seen in detailed analysis of the Supplementary Materials. Meanwhile, in order to compare the effect of annealing treatment on the HfOx-based device, the sulfur undoped devices at $400^{\circ} \mathrm{C}, 500{ }^{\circ} \mathrm{C}$, and $600^{\circ} \mathrm{C}$ were prepared and device performance was investigated, as shown in the Figure 2c. As a result, all of these devices show typical bipolar $\mathrm{I}-\mathrm{V}$ characteristics, and compared with the untreated device, the device performance was changed to some extent. However, the device performance was not as significantly improved as that of the S-doped device, which indicates that S-doping plays a key role in improving device performance compared with the annealing treatment of HfOx-based RRAM. Figure 3 shows the AFM of the undoped and S-doped HfOx films. The root mean square (RMS) of the S-doped films was enlarged, which may be caused by the deposition rate of sulfur ions increasing with rising temperature, and increased the interface contact area between the ITO and the dielectric layer (ITO is a good oxygen storage layer [26]). As shown in Figure 4a, the HRS/LRS ratio increased obviously. Figure $4 b, c$ shows probability distribution histograms of LRS and HRS, respectively. According to the Gaussian function, the statistical mean value and standard deviation (SD) are marked in the charts in Figure 4. The discrete coefficients (SD/mean ratio) of LRS (HRS) are $8.18 \%, 3.66 \%$, and $2.54 \%(14.05 \%$, $7.20 \%$, and $6.85 \%$ ) for D1, D2, and D3, respectively. A superior RS performance can be achieved with the $\mathrm{D} 3$ device. In addition, the endurance of $10^{6}$ alternate current (AC) cycles is shown in Figure S3 for D1, D2, and D3, respectively. For a comparison of the switching voltages, the cumulative distributions of $\mathrm{V}_{\text {SET }} / \mathrm{V}_{\text {RESET }}$ are presented in Figure 5a. According to the statistical voltage, the mean values of $\mathrm{V}_{\text {SET }}$ for D1, D2, and $\mathrm{D} 3$ are $+1.05 \mathrm{~V} /+0.65 \mathrm{~V} /+0.51 \mathrm{~V}$ and $\mathrm{SD}$ are $0.095 / 0.051 / 0.012$, respectively. The discrete coefficients of $\mathrm{V}_{\text {SET }}$ decreased from $9.05 \%$ to $2.35 \%$. In addition, the mean values of $\mathrm{V}_{\text {RESET }}$ for three devices are $-1.06 \mathrm{~V} /-0.78 \mathrm{~V} /-0.49 \mathrm{~V}$ and the $\mathrm{SD}$ values are $0.086 / 0.056 / 0.013$, respectively. The discrete coefficients decreased from $8.11 \%$ to $2.65 \%$. S-doping reduced the $\mathrm{V}_{\mathrm{SET}} / \mathrm{V}_{\mathrm{RESET}}$ and improved the uniformity of the switching voltages. Figure $5 \mathrm{~b}$ shows the retention characteristics of the S-doped device; the resistance values of both HRS and LRS exhibited robust stability and showed undetectable signs of degradation over $10^{4} \mathrm{~s}$ at $85^{\circ} \mathrm{C}$. Furthermore, in order to test the response speed of the devices, the SET/RESET pulses (width of $50 \mu$ s and amplitudes of $\pm 2 \mathrm{~V}, \pm 1 \mathrm{~V}, \pm 0.8 \mathrm{~V}$, respectively) were utilized to switch the resistance states, as illustrated in Figure $6 \mathrm{a}-\mathrm{c}$. The read pulses $(100 \mu \mathrm{s} /+0.1 \mathrm{~V})$ were input before and after the SET/RESET pulses to monitor the resistance switching. The D3 could be switched to LRS (HRS) at a fast speed of $6.25 \mu \mathrm{s}(7.50 \mu \mathrm{s})$ using the pulse pair $(50 \mu \mathrm{s} / \pm 0.8 \mathrm{~V})$, meanwhile, it consumes energy from the SET (RESET) process at $9.08 \mathrm{~nJ}$ $(6.72 \mathrm{~nJ})$. This can be calculated by using the formula:

$$
\mathrm{W}=\mathrm{V} \times \mathrm{I} \times \mathrm{T},
$$

where $\mathrm{V}$ is the applied voltage of the pulse, $\mathrm{I}$ is the response current, and $\mathrm{T}$ is the response time. In comparison, the SET/RESET speed of D1 is $16.56 \mu \mathrm{s} / 23.12 \mu \mathrm{s}$, and the energy is $224.2 \mathrm{~nJ} / 155.8 \mathrm{~nJ}$. A more explicit presentation of the D1 device is shown in Figure S4. S-doping is able to significantly improve the switching speed and achieve the low energy consumption in HfOx-based devices. Meanwhile, the resistive switching performance of 
undoped and S-doped HfOx-based devices are summarized in Table 1. The comparison of the effect of S-doping with other different doping elements for HfOx-based RRAM where their properties are simply summarized, is shown in Table S1.
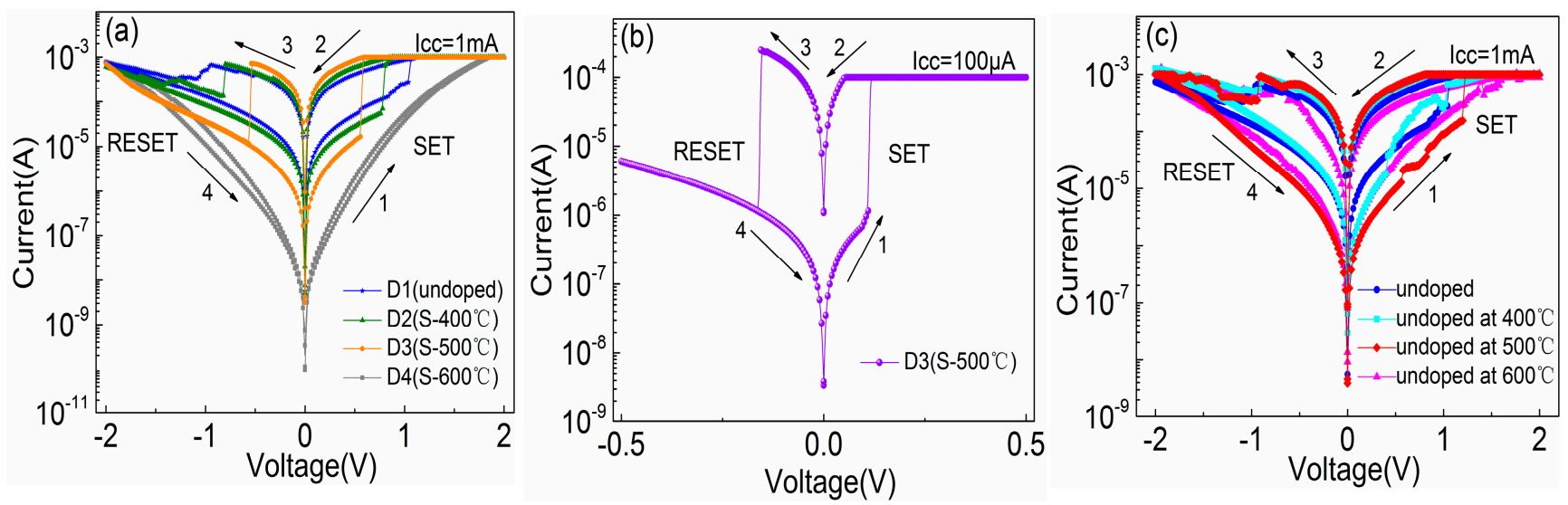

Figure 2. (a) Typical bipolar I-V cycles for DC sweep of the devices, (b) I-V cycle of D3 device under $100 \mu \mathrm{A}$ compliance current, (c) I-V cycles for the untreated and undoped at different temperatures annealing of the devices.
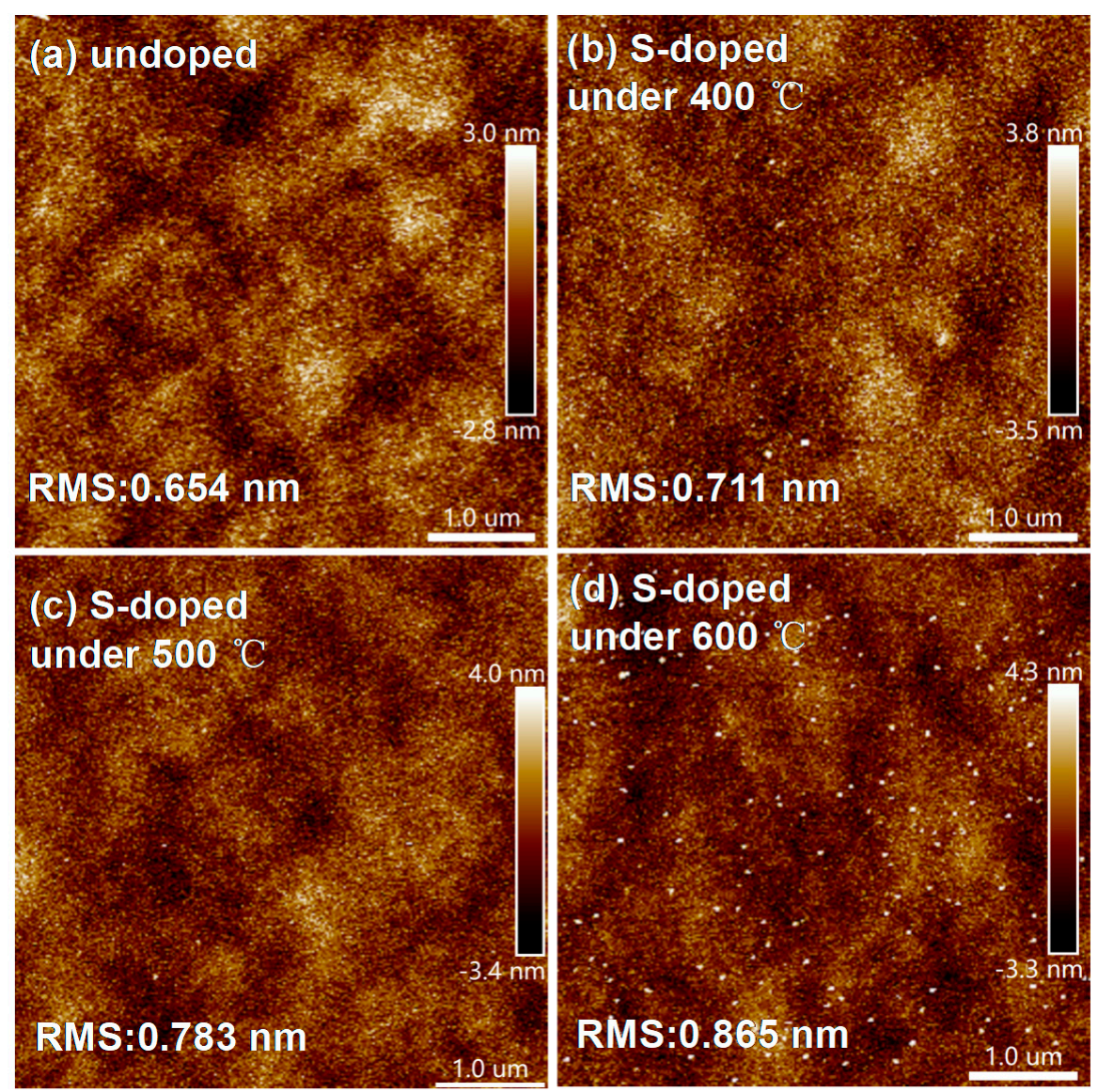

Figure 3. AFM images of the HfOx films of (a) undoped and S-doped at (b) $400{ }^{\circ} \mathrm{C}$, (c) $500{ }^{\circ} \mathrm{C}$, and (d) $600{ }^{\circ} \mathrm{C}$. 

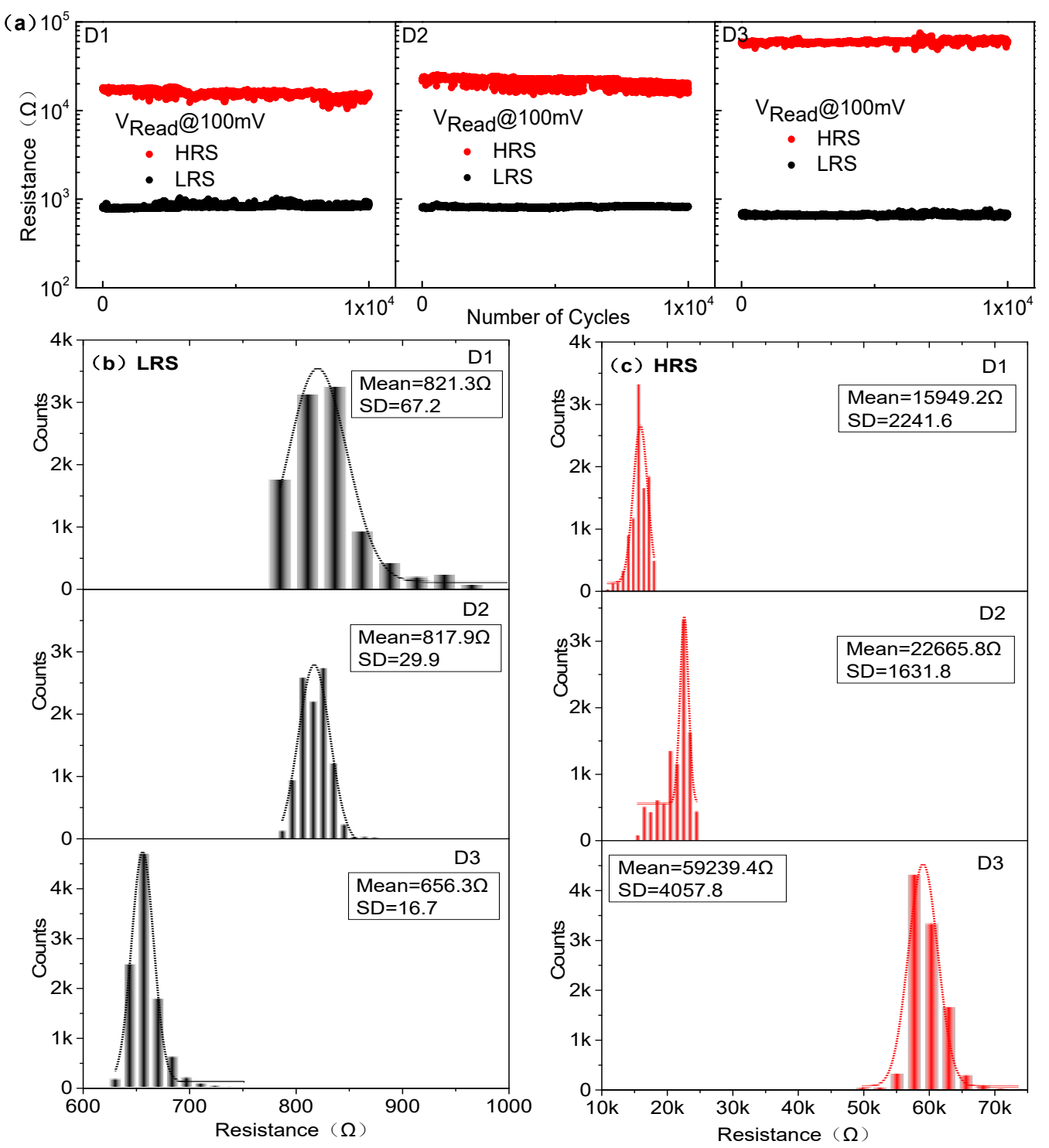

Figure 4. (a) Endurance of $10^{4}$ DC cycles of the D1, D2, and D3 devices, (b) Probability distribution histogram of (b) LRS and (c) HRS for the D1, D2, and D3 devices.
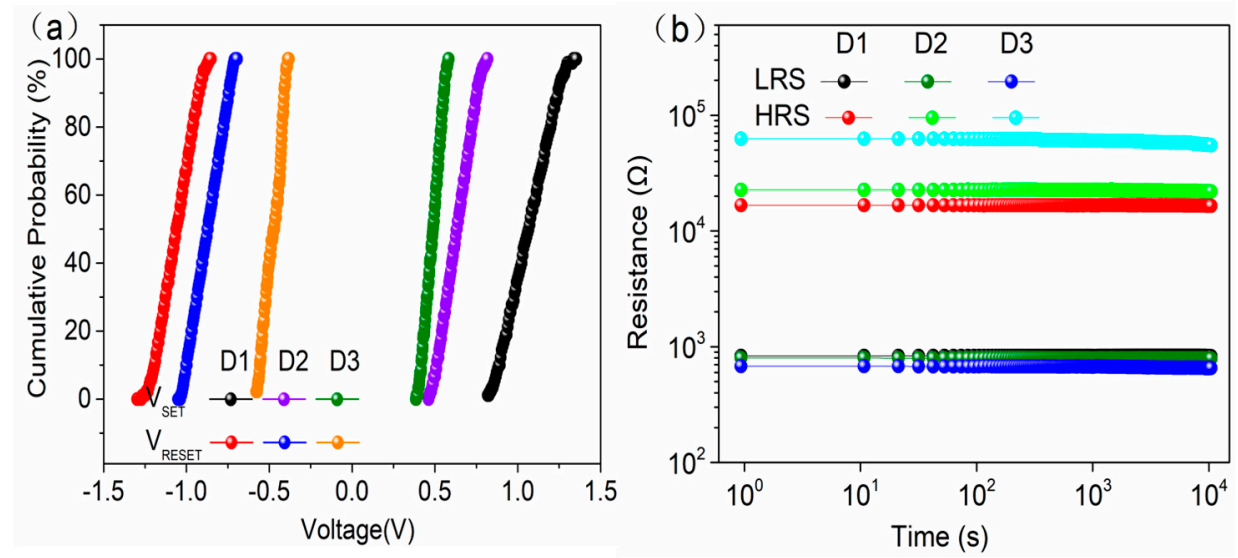

Figure 5. (a) Cumulative distributions of SET and RESET switching voltages for the D1, D2, and D3 devices, (b) Retention characteristics of the D1, D2, and D3 devices ( $\mathrm{V}_{\text {Read }}$ at $100 \mathrm{mV}$ for each device). 

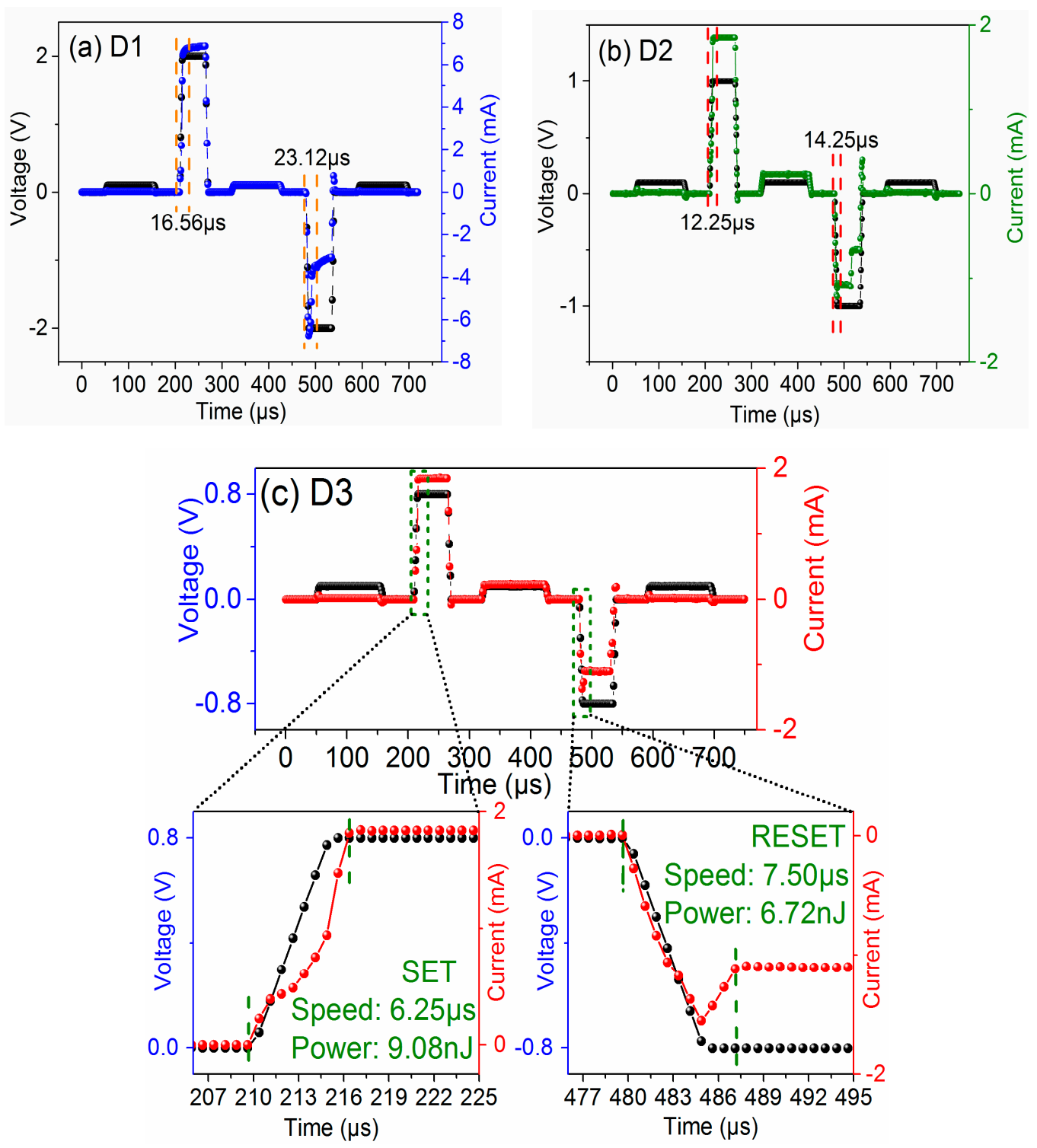

Figure 6. Switching speed of SET/RESET pulse pair for (a) D1, (b) D2, and (c) D3 devices. The black lines are the applied voltage pulses and the blue lines, green lines, and red lines are the separate current response pulses.

Table 1. Summarized the RS performance of undoped and S-doped HfOx-based devices.

\begin{tabular}{cccccccc}
\hline Devices & $\begin{array}{c}\mathbf{V}_{\text {forming }} \\
(\mathbf{V})\end{array}$ & $\begin{array}{c}\text { Voltage } \\
\mathbf{( V )}\end{array}$ & $\begin{array}{c}\text { HRS/LRS } \\
\text { Ratio }\end{array}$ & $\begin{array}{c}\text { Endurance } \\
\text { (AC cycles) }\end{array}$ & $\begin{array}{c}\text { Retention } \\
\text { at } 85^{\circ} \mathbf{C}(\mathbf{s})\end{array}$ & $\begin{array}{c}\text { Switching Speed } \\
(\mu \mathbf{s})\end{array}$ & $\begin{array}{c}\text { Energy Consumption } \\
(\mathbf{n J})\end{array}$ \\
\hline D1 & +2.18 & $+1.05 /-0.94$ & $\sim 12$ & $\begin{array}{c}10^{6} \\
\text { SET/RESET }\end{array}$ & $10^{4}$ & $\sim 16.56 / \sim 23.12$ & $224.2 / 155.8$ \\
D2 & +1.32 & $+0.76 /-0.78$ & $\sim 30$ & $\begin{array}{c}10^{6} \\
10^{6}\end{array}$ & $10^{4}$ & $\sim 12.25 / \sim 14.25$ & $22.54 / 15.25$ \\
D3 & +0.85 & $+0.11 /-0.15$ & $\sim 90$ & (Uniformity) & (Uniformity) & $\sim 6.25 / \sim 7.50$ & $9.08 / 6.72$ \\
D4 & - & $+2 /-2$ & $\sim 2$ & - & - & - & - \\
\hline
\end{tabular}

In order to clarify the effect of the S-doped HfOx device on the RS performance, the chemical composition of undoped and S-doped films was investigated using XPS. The binding energy was calibrated using the $\mathrm{C}-\mathrm{C}$ bonds of an adventitious $\mathrm{C}$ signal $(284.6 \mathrm{eV})$. Compared with the undoped film, the Hf $4 \mathrm{f}$ peaks of the S-doped film moved to a lower binding energy, indicating that sulfur ions incorporated into the HfOx films, as shown in Figure 7a. In Figure 7b, the S-doped films show a distinct peak of S 2p. XPS detected that the 
doping sulfur atomic percent content was $1.06 \mathrm{At} . \% / 1.66 \mathrm{At} . \%$ under $400{ }^{\circ} \mathrm{C} / 500{ }^{\circ} \mathrm{C}$. The peaks fitting of S 2 p spectra for S-doped of $500^{\circ} \mathrm{C}$ HfOx film are shown in Figure $7 \mathrm{c}$, and the binding energies of $S 2 \mathrm{p} 1 / 2$ and $S 2 \mathrm{p} 3 / 2$ are $165.3 \mathrm{eV}$ and $164.0 \mathrm{eV}$, respectively, consistent with the $\mathrm{S}^{2-}$ valence state. According to the NIST X-ray Photoelectron Spectroscopy Database [27] and the shift of the Hf $4 \mathrm{f}$ peaks, indicating that there new bonds were formed in the S-doped HfOx film. Interestingly, there is a weak peak of binding energy at $168.1 \mathrm{eV}$ and the corresponding valence state is $\mathrm{S}^{4+} / \mathrm{S}^{6+}$, which was caused by the impurity gas adsorbed onto the surface. Figure $7 \mathrm{~d}-\mathrm{f}$ shows the binding energies of the $\mathrm{O} 1 \mathrm{~s}$ peaks for undoped and S-doped films. In the $\mathrm{O} 1 \mathrm{~s}$ spectrum, the main peaks correspond to lattice oxygen at $529.8 \mathrm{eV}, 530.0 \mathrm{eV}$, and $530.1 \mathrm{eV}$ and the shoulder peaks correspond to non-lattice oxygen at $531.2 \mathrm{eV}, 531.3 \mathrm{eV}$ and $531.3 \mathrm{eV}$, respectively. Through the oxygen area percentages, the non-lattice oxygen components increased from $35.99 \%$ to $44.12 \%$ and $49.76 \%$, indicating that oxygen vacancy contents increased after S-doping. The increased oxygen vacancies were more beneficial in forming and rupturing the CFs in the switching process $[28,29]$. The XPS spectra of $\mathrm{Hf} 4 \mathrm{f}, \mathrm{S} 2 \mathrm{p}$ and O 1 s for S-doped of $600{ }^{\circ} \mathrm{C} \mathrm{HfOx} \mathrm{film}$ are shown in Figure S5. It is worth mentioning that the non-lattice oxygen component in the S-doped $600{ }^{\circ} \mathrm{C} \mathrm{HfOx}$ film was lower than that of the undoped film, which makes it harder for the D4 device to form oxygen vacancy type CFs. Afterwards, the conductive mechanisms were analyzed by the log-log linear fitting of LRS/HRS for D1, D2, and D3. Figure 8a shows the $\ln (\mathrm{I})-\ln (\mathrm{V})$ linear fitting of LRS after SET. All the fitting slopes are $\sim 1$, indicating the Ohmic conduction mechanism in LRS. Figure 8b shows the ln (I)-sqrt (V) linear fitting of HRS after RESET, which proved to be consistent with the Schottky emission mechanism. Figure S6 shows that the Schottky emission mechanism was confirmed by variable temperature experiments.
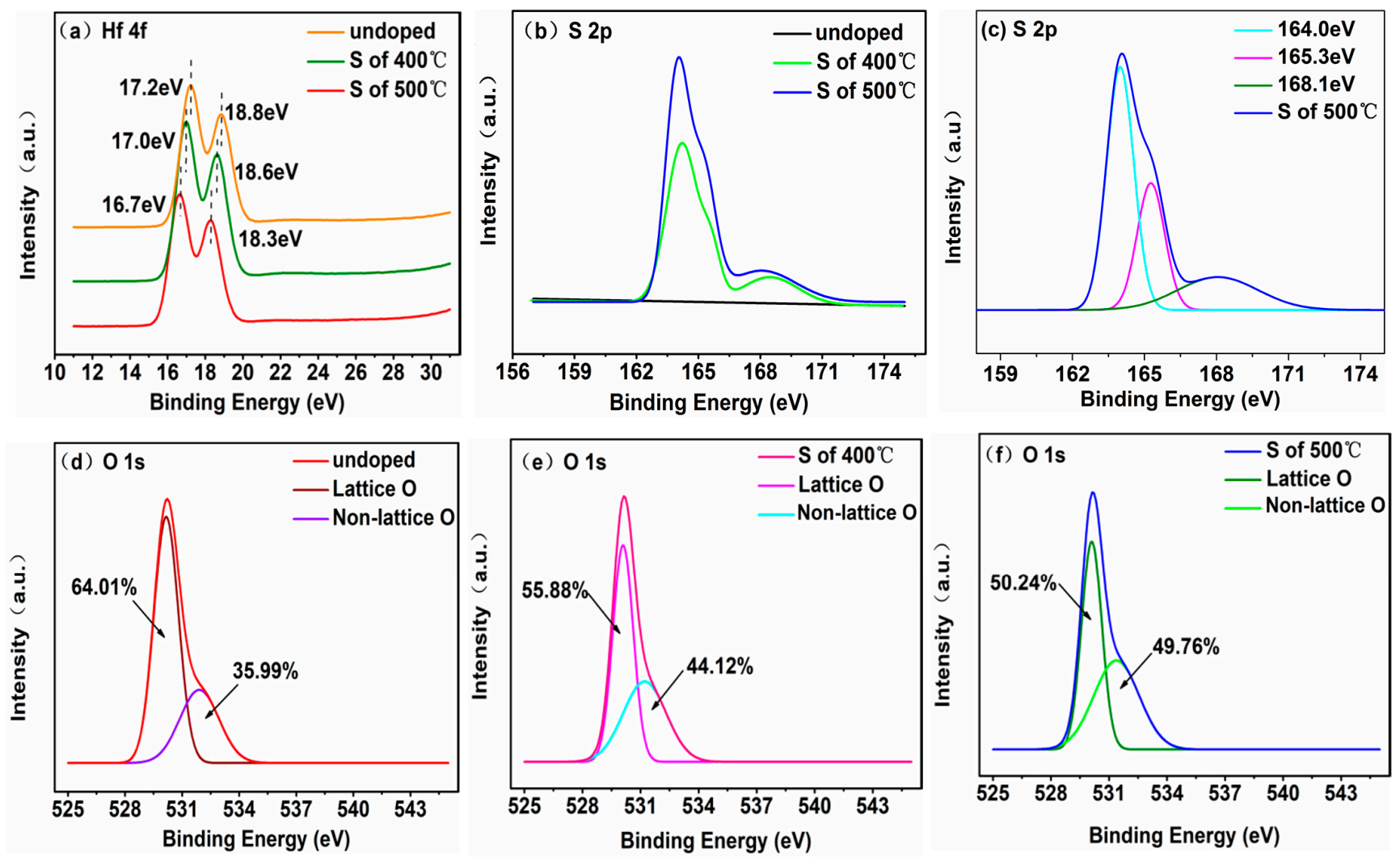

Figure 7. XPS spectra of (a) Hf 4f, (b,c) S 2p, (d-f) O 1s for the undoped and S-doped HfOx films. 

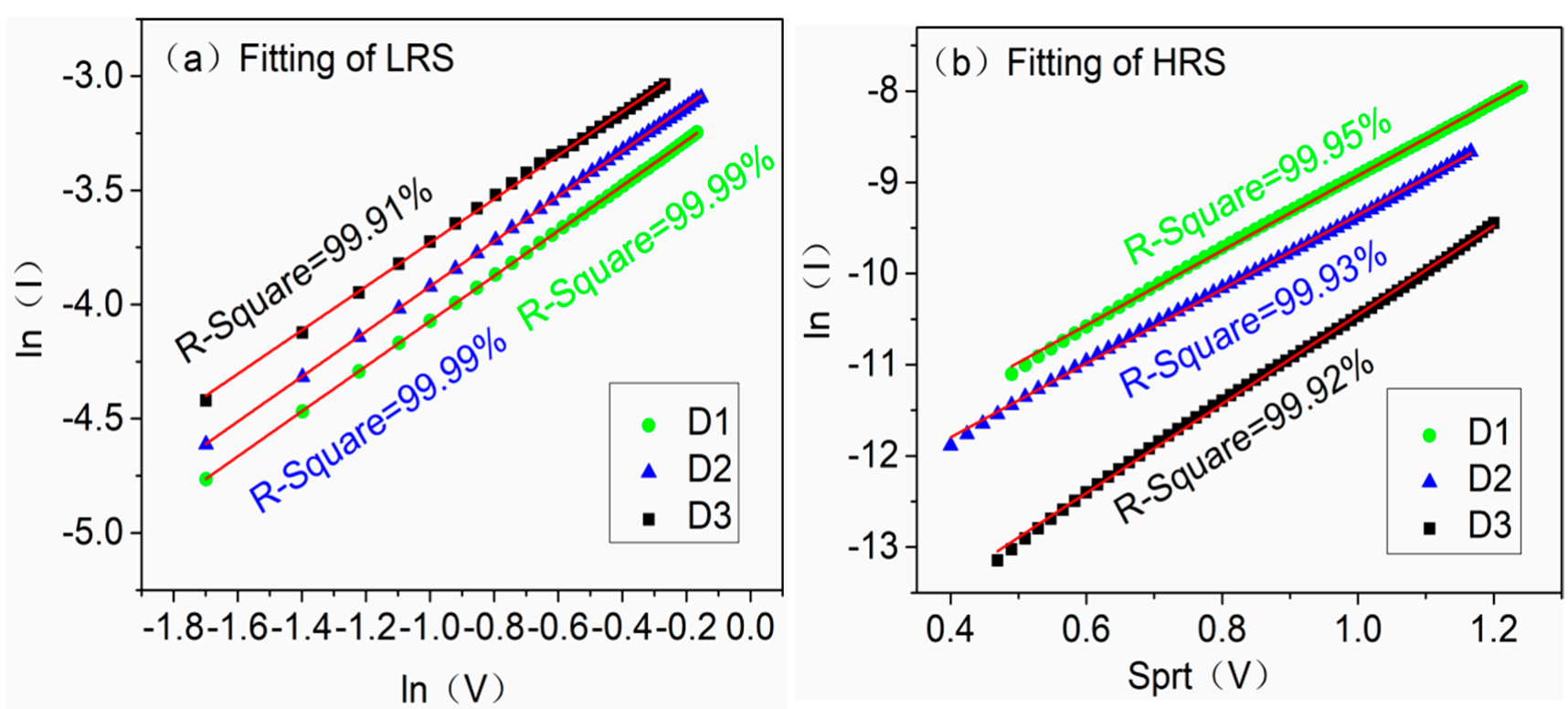

Figure 8. For the D1, D2, and D3 devices: (a) linear fit of LRS, (b) linear fit of HRS. (R-squared values are all greater than $99.9 \%$, and are marked in the figure).

Based on XPS data and the fitting of the LRS/HRS conduction behavior, a switching mechanism was considered, as shown schematically in Figure 9a,b. The RS mechanism could be explained by the formation and rupturing of CFs, consistent with oxygen vacancies [30]. In the D1 device, the interstitial oxygen ions migrated toward TE under positive voltage. Moreover, the movement of oxygen ions was affected by joule heat and diffused around the film [31]. While TE was applied with a negative voltage, oxygen ions injected $\mathrm{HfOx}$ film from TE and combined with oxygen vacancies to break CFs. More frequently in the switching process, the partial oxygen ions break away from the modulation of the electric field and become unevenly distributed, which make the formed CFs unstable and random. The formation and rupture of CFs was more uniform in the D3 device. Oxygen ions moved in the same direction as the $\mathrm{D} 1$ device at positive and negative voltages. The lattice gaps of the HfOx film became larger because of the different bonding of Hf-S and $\mathrm{Hf}-\mathrm{O}$ (the radius of the oxygen ion was $1.4 \AA$ and that of the sulfur ion was $1.84 \AA$ [32]), thus interstitial oxygen ions moved more easily in the gaps after S-doping. Furthermore, XPS proves that S-doping introduces more oxygen vacancies into HfOx film, which causes the S-doped HfOx film to be in a state of oxygen deficiency, and higher oxygen deficiency will favor a better performance for the device [31]. Furthermore, oxygen vacancies easily generate around $\mathrm{S}$ dopants to suppress the random formation of CFs [33,34], thus improving performance in the TiN/HfOx/ITO device. 

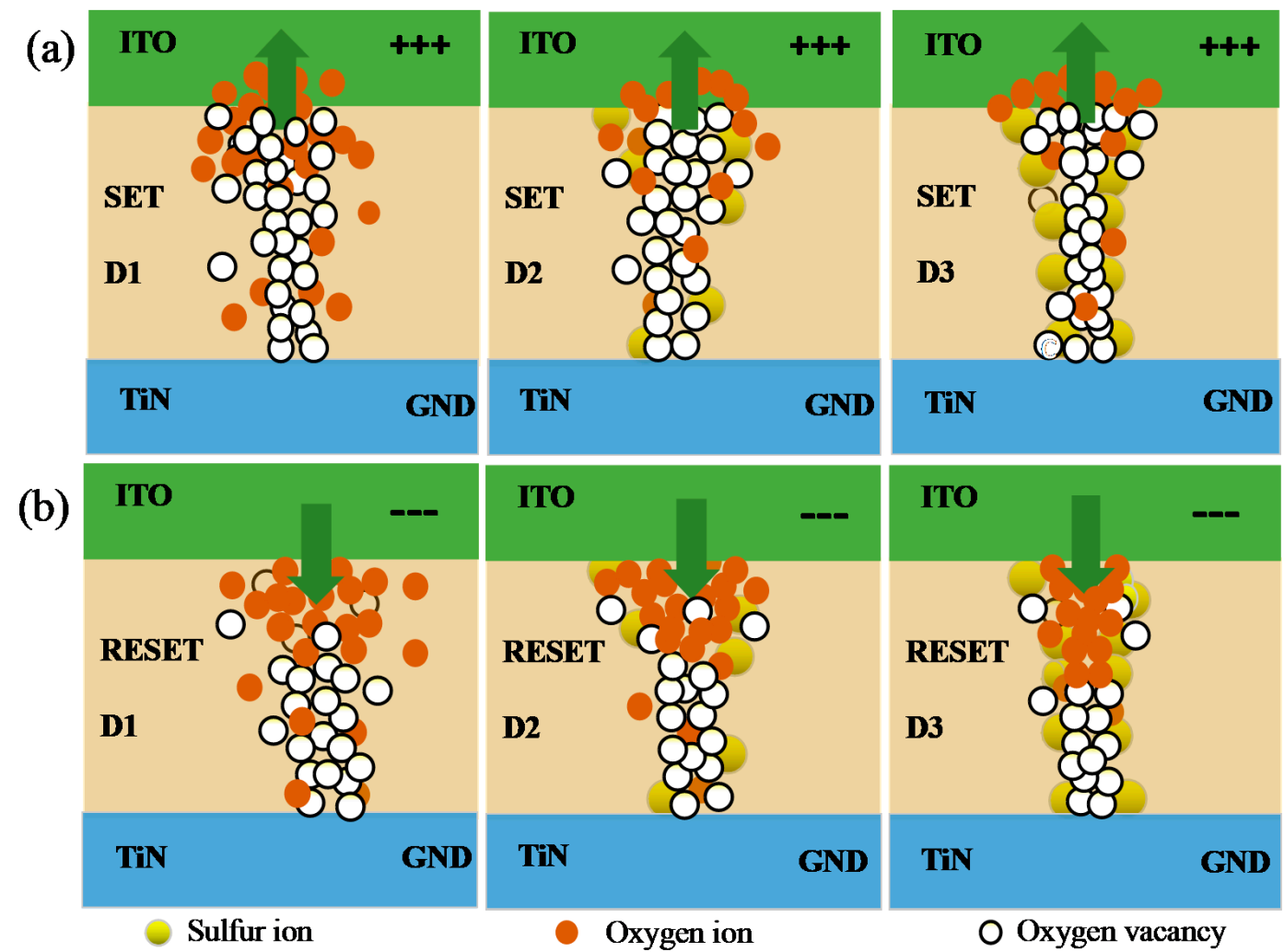

Figure 9. Schematic diagram of the switching mechanism of SET process for (a) D1, D2, and D3 and the RESET process for (b) D1, D2, and D3. (The green arrow represents the movement direction of oxygen ions, and the green box is the top electrode ITO of the device, and the blue box is the bottom electrode GND).

\section{Conclusions}

In summary, S-doped HfOx RRAM devices were fabricated using APCVD treatment. The electrical performances are enhanced for the S-doped HfOx device, and show high uniformity, lower switching voltages, lager HRS/LRS ratio, stable endurance, long data retention characteristics, fast response speed, and low energy consumption of SET/RESET. Based on the experimental results, the improvements of the memory performance could be attributed to the S-doping effect in the HfOx-based RRAM. The results identify that Sdoping increases oxygen vacancies in the HfOx-based device and could effectively suppress the randomness of oxygen vacancy $\mathrm{CF}$ formation. The results also demonstrate that the S-doped technology has potential applications in other oxide-based RRAMs.

Supplementary Materials: The following are available online at https://www.mdpi.com/article/ 10.3390/ma14123330/s1, Figure S1: The forming process of (a) D1, (b) D2, (c) D3, (d) D4 and the consecutive I-V cycles of (e) D1, (f) D2, (g) D3, respectively. Figure S2: The I-V curves of each device under $100 \mu \mathrm{A}$ and $1 \mathrm{~mA}$ compliance current. Figure S3: The endurance of $10^{6} \mathrm{AC}$ cycles for D1, D2, D3 devices, respectively. Figure S4: The switching speed upon SET/RESET pulse pair for (a) D1 and (b) D2. Figure S5: XPS spectra of (a) Hf 4f, (b) S 2p, (c) O 1s for S-doped of $600{ }^{\circ} \mathrm{C}$ HfOx film. Figure S6: The Schottky emission fitting for (a) D1, (b) D2, (c) D3 devices at temperature ranged from $300 \mathrm{~K}$ to $340 \mathrm{~K}$. Table S1: Summary of the properties of HfOx-based RRAM for different doping elements.

Author Contributions: Conceptualization, Z.Z. and K.Z.; methodology, Z.Z.; software, Y.S.; validation, K.Z.; formal analysis, F.W.; investigation, Z.Z.; resources, K.Z.; data curation, F.W. and K.H.; writing-original draft preparation, Z.Z.; writing-review and editing, F.W.; visualization, K.H.; supervision, S.S. and Z.S.; funding acquisition, K.Z. All authors have read and agreed to the published version of the manuscript. 
Funding: This work was supported by the National Key Research and Development Program of China (Grant No.2017YFB0405600), Natural Science Foundation of Tianjin City (Grant Nos.18JCZDJC30500, 17JCYBJC16100 and 17JCQNJC00900) and National Natural Science Foundation of China (Grant Nos.61404091, 61274113, 61505144, 51502203 and 51502204) and Open Project of State Key Laboratory of Functional Materials for Information (SKL202007).

Institutional Review Board Statement: Not applicable.

Informed Consent Statement: Not applicable.

Data Availability Statement: Data available on request. The data presented in this study are available on request from the corresponding author.

Conflicts of Interest: The authors declare no conflict of interest.

\section{References}

1. Han, S.-T.; Zhou, Y.; Roy, V.A.L. Towards the Development of Flexible Non-Volatile Memories. Adv. Mater. 2013, 25, 5425-5449. [CrossRef]

2. Lee, J.; Schell, W.; Zhu, X.; Kioupakis, E.; Lu, W.D. Charge Transition of Oxygen Vacancies during Resistive Switching in Oxide-Based RRAM. ACS Appl. Mater. Interfaces 2019, 11, 11579-11586. [CrossRef] [PubMed]

3. Chen, X.; Feng, J.; Bae, D. Drastic reduction of RRAM reset current via plasma oxidization of TaOx film. Appl. Surf. Sci. 2015, 324, 275-279. [CrossRef]

4. Ku, B.; Abbas, Y.; Sokolov, A.S.; Choi, C. Interface engineering of ALD HfO2-based RRAM with Ar plasma treatment for reliable and uniform switching behaviors. J. Alloy. Compd. 2018, 735, 1181-1188. [CrossRef]

5. Tan, T.; Guo, T.; Liu, Z. Au doping effects in HfO2-based resistive switching memory. J. Alloy. Compd. 2014, 610, 388-391. [CrossRef]

6. Wu, Q.; Banerjee, W.; Cao, J.; Ji, Z.; Li, L.; Liu, M. Improvement of durability and switching speed by incorporating nanocrystals in the HfOx based resistive random access memory devices. Appl. Phys. Lett. 2018, 113, 023105. [CrossRef]

7. Michalas, L.; Stathopoulos, S.; Khiat, A.; Prodromakis, T. Conduction mechanisms at distinct resistive levels of Pt/TiO2-x/Pt memristors. Appl. Phys. Lett. 2018, 113, 143503. [CrossRef]

8. Zhuge, F.; Peng, S.; He, C.; Zhu, X.; Chen, X.; Liu, Y.; Li, R.-W. Improvement of resistive switching in Cu/ZnO/Pt sandwiches by weakening the randomicity of the formation/rupture of Cu filaments. Nanotechnology 2011, 22, 275204. [CrossRef]

9. He, P.; Ye, C.; Wu, J.; Wei, W.; Wei, X.; Wang, H.; Zhang, R.; Zhang, L.; Xia, Q.; Wang, H. Effect of sputtering atmosphere on the characteristics of $\mathrm{ZrOx}$ resistive switching memory. Semicond. Sci. Technol. 2017, 32, 055016. [CrossRef]

10. Lee, M.-J.; Lee, C.B.; Lee, D.; Lee, S.R.; Chang, M.; Hur, J.H.; Kim, Y.-B.; Kim, C.-J.; Seo, D.H.; Seo, S.; et al. A fast, high-endurance and scalable non-volatile memory device made from asymmetric Ta2O5-x/TaO2-x bilayer structures. Nat. Mater. 2011, 10, 625-630. [CrossRef] [PubMed]

11. Lin, Y.; Zeng, T.; Xu, H.; Wang, Z.; Zhao, X.; Liu, W.; Ma, J.; Liu, Y. Transferable and Flexible Artificial Memristive Synapse Based on WO x Schottky Junction on Arbitrary Substrates. Adv. Electron. Mater. 2018, 4, 1-8. [CrossRef]

12. Xie, H.; Liu, Q.; Li, Y.; Lv, H.; Wang, M.; Liu, X.; Sun, H.; Yang, X.; Long, S.; Liu, S.; et al. Nitrogen-induced improvement of resistive switching uniformity in a HfO2-based RRAM device. Semicond. Sci. Technol. 2012, 27, 125008. [CrossRef]

13. Chang, T.-C.; Chang, K.-C.; Tsai, T.-M.; Chu, T.-J.; Sze, S.M. Resistance random access memory. Mater. Today 2016, 19, 254-264. [CrossRef]

14. Wong, H.-S.P.; Lee, H.-Y.; Yu, S.; Chen, Y.-S.; Wu, Y.; Chen, P.-S.; Lee, B.; Chen, F.T.; Tsai, M.-J. Metal-Oxide RRAM. Proc. IEEE Inst. Electr. Electron Eng. 2012, 100, 1951-1970. [CrossRef]

15. Sokolov, A.S.; Son, S.K.; Lim, D.; Han, H.H.; Jeon, Y.-R.; Lee, J.H.; Choi, C. Comparative study of Al2O3, HfO2, and HfAlOxfor improved self-compliance bipolar resistive switching. J. Am. Ceram. Soc. 2017, 100, 5638-5648. [CrossRef]

16. Qi, M.; Cao, S.; Yang, L.; You, Q.; Shi, L.; Wu, Z. Uniform multilevel switching of graphene oxide-based RRAM achieved by embedding with gold nanoparticles for image pattern recognition. Appl. Phys. Lett. 2020, 116, 163503. [CrossRef]

17. Tang, L.; Maruyama, H.; Han, T.; Nino, J.C.; Chen, Y.; Zhang, D. Resistive switching in atomic layer deposited HfO2/ZrO2 nanolayer stacks. Appl. Surf. Sci. 2020, 515, 146015. [CrossRef]

18. Kim, S.; Abbas, Y.; Jeon, Y.-R.; Sokolov, A.S.; Ku, B.; Choi, C. Engineering synaptic characteristics of TaOx/HfO2 bi-layered resistive switching device. Nanotechnology 2018, 29, 415204. [CrossRef] [PubMed]

19. Zhang, K.; Sun, K.; Wang, F.; Han, Y.; Jiang, Z.; Zhao, J.; Wang, B.; Zhang, H.; Jian, X.; Wong, H.-S.P.; et al. Ultra-Low Power $\mathrm{Ni} / \mathrm{HfO}$ /TiOx/TiN Resistive Random Access Memory With Sub-30-nA Reset Current. IEEE Electron. Device Lett. 2015, 36, 1018-1020. [CrossRef]

20. Sun, C.; Lu, S.; Jin, F.; Mo, W.; Song, J.; Dong, K. Control the switching mode of Pt/HfO2/TiN RRAM devices by tuning the crystalline state of TiN electrode. J. Alloy. Compd. 2018, 749, 481-486. [CrossRef]

21. Lin, C.-Y.; Chen, P.-H.; Chang, T.-C.; Chang, K.-C.; Zhang, S.-D.; Tsai, T.-M.; Pan, C.-H.; Chen, M.-C.; Po-Hsun, C.; Tseng, Y.-T.; et al. Attaining resistive switching characteristics and selector properties by varying forming polarities in a single HfO2-based RRAM device with a vanadium electrode. Nanoscale 2017, 9, 8586-8590. [CrossRef] [PubMed] 
22. Tan, T.; Guo, T.; Chen, X.; Li, X.; Liu, Z. Impacts of Au-doping on the performance of Cu/HfO2/Pt RRAM devices. Appl. Surf. Sci. 2014, 317, 982-985. [CrossRef]

23. Jang, J. Effect of electrode material on characteristics of non-volatile resistive memory consisting of Ag2S nanoparticles. AIP Adv. 2016, 6, 75006. [CrossRef]

24. Li, N.; Wang, Y.; Sun, H.; Hu, J.; Zheng, M.; Ye, S.; Wang, Q.; Li, Y.; He, D.; Wang, J.; et al. Resistive switching behaviors and mechanisms of HfS2 film memory devices studied by experiments and density functional theory calculations. Appl. Phys. Lett. 2020, 116, 063503. [CrossRef]

25. Zheng, J.; Cheng, B.; Wu, F.; Su, X.; Xiao, Y.; Guo, R.; Lei, S. Modulation of Surface Trap Induced Resistive Switching by Electrode Annealing in Individual PbS Micro/Nanowire-Based Devices for Resistance Random Access Memory. ACS Appl. Mater. Interfaces 2014, 6, 20812-20818. [CrossRef]

26. Chen, P.-H.; Chang, K.-C.; Chang, T.-C.; Tsai, T.-M.; Pan, C.-H.; Chu, T.-J.; Chen, M.-C.; Huang, H.-C.; Lo, I.; Zheng, J.-C.; et al. Bulk Oxygen-Ion Storage in Indium-Tin-Oxide Electrode for Improved Performance of HfO2-Based Resistive Random Access Memory. IEEE Electron. Device Lett. 2016, 37, 280-283. [CrossRef]

27. Naumkin, A.V.; Kraut-Vass, A.; Gaarenstroom, S.W.; Powell, C.J. NIST X-Ray Photoelectron Spectroscopy (XPS) Database. 2012. Available online: https://srdata.nist.gov/xps/selEnergyType.aspx (accessed on 25 March 2021).

28. Qi, M.; Tao, Y.; Wang, Z.; Xu, H.; Zhao, X.; Liu, W.; Ma, J.; Liu, Y. Highly uniform switching of HfO2-x based RRAM achieved through Ar plasma treatment for low power and multilevel storage. Appl. Surf. Sci. 2018, 458, 216-221. [CrossRef]

29. Tan, T.; Du, Y.; Cao, A.; Sun, Y.; Zha, G.; Lei, H.; Zheng, X. The resistive switching characteristics of Ni-doped HfO film and its application as a synapse. J. Alloy. Compd. 2018, 766, 918-924. [CrossRef]

30. Balatti, S.; Larentis, S.; Gilmer, D.C.; Ielmini, D. Multiple Memory States in Resistive Switching Devices through Controlled Size and Orientation of the Conductive Filament. Adv. Mater. 2013, 25, 1474-1478. [CrossRef]

31. Clima, S.; Govoreanu, B.; Jurczak, M.; Pourtois, G. HfOx as RRAM material - First principles insights on the working principles. Microelectron. Eng. 2014, 120, 13-18. [CrossRef]

32. Dean, J.A. Lange's Handbook of Chemistry, 13th ed.; McGraw-Hill Book Company: New York, NY, USA, 1985.

33. Guo, T.; Tan, T.; Liu, Z.; Liu, B. Oxygen vacancy modulation and enhanced switching behavior in HfOx film induced by Al doping effect. J. Alloy. Compd. 2016, 686, 669-674. [CrossRef]

34. Wang, M.-H.; Chang, T.-C.; Shih, C.-C.; Tseng, Y.-T.; Tsai, T.-M.; Zheng, H.-X.; Wu, P.-Y.; Huang, H.-C.; Chen, W.-C.; Huang, J.-W.; et al. Performance improvement after nitridation treatment in HfO2-based resistance random-access memory. Appl. Phys. Express 2018, 11, 084101. [CrossRef] 